\title{
Lista de Revisores / List of reviewers 2016
}

\author{
Albay, Soner (Turquía) \\ Algieri, Rubén D. (Argentina) \\ Amer, Mariano A. R. (Argentina) \\ Apaydin, Nihal (Turquía) \\ Armand Ugón, Gustavo (Uruguay) \\ Arraez-Aybar, Luis A. (España) \\ Baca, Vaclav (República Checa) \\ Barbeito, Claudio G. (Argentina) \\ Barut, Cagatay (Turquía) \\ Battaglia, Patrick J. (Estados Unidos) \\ Brahim, Manuel (Argentina) \\ Cabanillas Padilla, Juan (Perú) \\ Campero, A. Álvaro (Argentina) \\ Cárdenas Valenzuela, Julio (Chile) \\ Cömert, Ayhan (Turquía) \\ Corbera, Juan Alberto (España) \\ Criado del Río, Luis E. (Argentina) \\ David, Oscar P. (Argentina) \\ de la Cruz- Sanchez, Ernesto (España) \\ Elizondo-Omaña, Rodrigo E. (México) \\ Espósito, Evangelina (Canadá) \\ Felipe, Antonio E. (Argentina) \\ Forero Bulla, Germán (Colombia) \\ Garud, Rajendra S. (India) \\ Georgiev, Georgi P. (Bulgaria) \\ Hassan, Ana (Argentina) \\ Haviarová, Zora (Eslovaquia) \\ Holda, Mateusz (Polonia) \\ Khan, Aaijaz A. (Malasia)
}

Khayrulin, Radik (Rusia)

Loster, Jolanta E. (Polonia)

Madhankumar, S. J. (India)

Mahakkanukrauh, Pasuk (Tailandia)

Martínez, Fernando (Uruguay)

Martínez Soriano, Francisco (España)

Martínez-González, J. María (España)

Martino, Elia del C. (Argentina)

Marzola, Clovys (Brasil)

Matsutani, Shinji (J apón)

Matveeva, Niki (Macedonia)

Medina Ruiz, Blas A. (Paraguay)

Mompeó Corredera, Blanca R. (España)

Murlimanju, Bukkambudhi V. (India)

Natsis, Konstantinos (Grecia)

Nikolova, Silvia (Bulgaria)

Olate, Sergio (Chile)

Olivera, Eduardo (Uruguay)

Polguj, Michal (Polonia)

Prada Oliveira, Arturo (España)

Prat, Guillermo D. (Argentina)

Reblet, Concepción (España)

Reina, Miguel A. (España)

Rodríguez Baeza, Alfonso (España)

Russo, Alejandro M. (Uruguay)

Sañudo, José R. (España)

Sforza, Chiarella (Italia)

Soames, Roger W. (Reino Unido)

Tamayo, Sergio (Argentina) 
Teofilovski-Parapid, Gordana (Serbia)

Tohno, Yoshiyuki (Tailandia)

Toledo Neto, Joao L. (Brasil)

Tosovska Lazarova, Dobrila (Macedonia)
Totlis, Trifon (Grecia)

Vázquez-Osorio, Teresa (España)

Zahoi, Delia E. (Rumania) 